\title{
Estudo retrospectivo de recidiva da hanseníase no Estado do Espírito Santo
}

\author{
Retrospective study on leprosy relapse in the \\ State of Espírito Santo
}

\author{
Lucia Martins Diniz ${ }^{1,3}$, Marilda Vieira Moreira ${ }^{2}$, Marizete Altoé Puppin ${ }^{2}$ \\ e Maria Leide Wand Del Rey de Oliveira ${ }^{3}$
}

\begin{abstract}
RESUMO
Recidiva de hanseníase é o reaparecimento da doença, após tratamento regular com os esquemas vigentes e alta por cura. Neste estudo de coorte retrospectivo e descritivo o objetivo foi avaliar as características dos casos de recidiva de hanseníase no Estado do Espírito Santo entre 2000 e 2005. As estratégias de investigação foram: monitoramento das entradas no SINAN, análise das fichas e discussão dos casos no Centro de Referência Estadual. Foram estudados 104 casos de recidiva, representando 1,12\% em relação aos casos novos diagnosticados no período. A maior frequiência foi entre 21 a 60 anos; 59,6\% eram do sexo masculino; 44,2\% apresentaram a recidiva após cinco anos da alta; 66,4\% eram multibacilares, sendo 42,2\% com baciloscopias positivas (bacilos íntegros), portanto recidivas. Baciloscopias negativas foram observadas em $57,8 \%$. Estudos prospectivos devem ser feitos para estabelecimento da taxa real de recidiva.
\end{abstract}

Palavras-chaves: Hanseníase. Recorrência. Recidiva. Epidemiologia. Poliquimioterapia.

\begin{abstract}
Leprosy relapse is the reappearance of the disease after regular treatment with current regimens and discharge due to cure. In this retrospective and descriptive cohort study, the aim was to evaluate the characteristics of cases of leprosy relapse in the State of Espírito Santo between 2000 and 2005. The investigation strategies consisted of monitoring the SINAN entries, with file analysis and case discussions at the State Reference Center. One hundred and four cases of relapse were studied, representing $1.12 \%$ of the new cases detected over this period. The greatest frequency was between 21 and 60 years of age; $59.6 \%$ were men; $44.2 \%$ presented relapse more than five years after discharge; $66.4 \%$ were multibacillary; and $42.2 \%$ presented positive bacilloscopy (complete bacilli) and therefore were relapse cases. Negatives bacilloscopy was observed in $57.8 \%$. Prospective studies should be conducted to establish the real relapse rate.
\end{abstract}

Key-words: Leprosy. Recurrence. Relapse. Epidemiology. Multidrug therapy.

Caso de recidiva na hanseníase é o paciente que tratado regularmente pelos esquemas oficiais vigentes no país, recebe alta por cura, e após um período de incubação variável aparece com sinais e sintomas de atividade da doença ${ }^{14}$.

$\mathrm{Na}$ recidiva, ocorre o aparecimento de novas lesões características das formas clínicas da hanseníase, nos mesmos locais do primeiro diagnóstico ou em outras áreas da pele e/ ou acometimento de nervos periféricos (neurite). Nos casos paucibacilares (PB), é imprescindível o diagnóstico diferencial com a reação reversa, sendo que esta responde favoravelmente

\footnotetext{
1. Serviço de Dermatologia Santa Casa de Misericórdia de Vitória, ES. 2. Programa de Controle da Hanseníase da Secretaria de Estado da Saúde do Espírito Santo, Vitória, ES. 3. Serviço de Dermatologia Universidade Federal do Rio de Janeiro, Rio de Janeiro, RJ.

Endereço para correspondência: Dra. Lucia Martins Diniz. Praça San Martin 56/sala 405, Bairro Praia do Canto, 29055-170 Vitória, ES.

Tel: $55273325-0940$

e-mail:1diniz@viprede.com.br

Recebido para publicação em 22/01/2009

Aceito em 03/08/3009
}

com a terapia de corticosteróide sistêmico em doses preconizadas, diferente da recidiva ${ }^{14}$.

Também, suspeita-se de recidiva, quando os casos multibacilares (MB) iniciam reações após três anos da alta por cura ou que as apresentem durante o tratamento e permanecem desta maneira por cinco anos ${ }^{14}$.

Os fatores relacionados ao desenvolvimento da recidiva na hanseníase podem dever-se à persistência ou resistência do bacilo, à imunossupressão, gravidez, formas virchowianas avançadas do hospedeiro, à endemicidade do meio (reinfecção) e ao diagnóstico tardio, terapêutica inadequada ou irregular da doença e erro de classificação $0^{411} 17$.

O diagnóstico clínico deve ser acompanhado pela realização de baciloscopias, estudo histopatológico da lesão e se possível, a inoculação de bacilos no coxim plantar de camundongo ou a realização de técnicas moleculares, como a reação em cadeia da polimerase e o sequeciamento de regiões do DNA do Mycobacterium leprae, que são alvos de drogas, para a verificação de resistência medicamentosa, no material da lesão obtido por biópsia ${ }^{51420}$. 
0 período de incubação de recidiva da hanseníase obedece a linha de sugestão de Pattyn, sendo bifásica, onde as recidivas precoces resultam de terapia inadequada e as recidivas tardias se originam de reativação de organismos persistentes ou de reinfecções ${ }^{7}$. Os pacientes $\mathrm{PB}$ normalmente recidivam após um ano da alta por cura e os MB após cinco anos, sendo que os doentes de maior risco são os que apresentam maior carga bacilar, ou seja, índice baciloscópico inicial maior ou igual a quatro e final maior que dois ou três ${ }^{6}{ }^{16}$.

Estudos de avaliação de recidiva da hanseníase não adotam uniformidade no cálculo da taxa de recidiva, sendo a taxa pessoas-ano de recidiva, que é calculada pelo número de recidivas multiplicado por 1.000 e dividido pelo número de pessoas ano de acompanhamento, a que melhor reflete esta realidade, sendo aceitável a taxa de até $5 \%$ de recidiva ${ }^{317}$.

Os pacientes com recidiva são retratados com a poliquimioterapia (PQT) padrão, de acordo com a classificação clínica e laboratorial da hanseníase ${ }^{14}$.

O Estado do Espírito Santo encerrou o ano de 2005 com taxa de prevalência de 3,87 casos por 10.000 habitantes e taxa de detecção de 4,44 casos por 10.000 habitantes, a mais alta da região Sudeste. A proporção de cura entre os casos $\mathrm{PB}$ e $\mathrm{MB}$ vem aumentando nos últimos cinco anos. No ano 2000, estes percentuais foram para os $\mathrm{PB} 86,8 \%$ e para os MB $82,2 \%$. Em 2005 , estas taxas foram, respectivamente, de $91,5 \%$ e $86,4 \%$. 0 abandono de tratamento foi baixo, com média de $4,5 \%$ para 0 período $^{19}$

0 estudo teve por objetivo determinar a magnitude e as características clínicas e laboratoriais das recidivas da hanseníase no Estado do Espírito Santo, de maneira retrospectiva, durante o período de janeiro de 2000 a dezembro de 2005.

\section{PACIENTES E MÉTODOS}

Estudo de coorte, retrospectivo, descritivo, no qual foram adotadas as seguintes estratégias de investigação dos casos de recidiva no Estado do Espírito Santo, notificados durante o período de janeiro de 2000 a dezembro de 2005: a) levantamento (identificação) de todos os casos diagnosticados como hanseníase no período; b) identificação e reavaliação dos casos notificados como recidiva no Sistema de Informação de Agravos e Notificação (SINAN), com revisão dos prontuários do tratamento, após o primeiro diagnóstico; c) encaminhamento das fichas do SINAN e dos prontuários ao Centro de Referência Estadual em HanseníaseSanta Casa de Misericórdia de Vitória - Espírito Santo para análise detalhada, reavaliação e conclusão dos diagnósticos de recidiva; d) dos casos incluídos no estudo como recidiva de hanseníase, foram analisadas as seguintes variáveis: idade, sexo, período de incubação da recidiva, forma clínica, índice baciloscópico e terapêuticas no primeiro diagnóstico e na recidiva, e no caso desta, o número de lesões e de troncos nervosos afetados e 0 grau de incapacidade.

Os critérios de inclusão no estudo foram: os pacientes notificados com o diagnóstico clínico de recidiva de hanseníase na ficha do SINAN, que haviam sido tratados para esta doença, de forma regular, recebido alta por cura e que apareceram com sinais clínicos e/ou laboratoriais de atividade da doença.

Os critérios de exclusão foram: período de incubação da recidiva inferior a um ano, erro de diagnóstico, irregularidade no primeiro tratamento e descrição de lesões compatíveis com reação de hanseníase ou outro diagnóstico dermatológico.

Após a conclusão de recidiva, realizou-se o cálculo da magnitude da recidiva entre os casos que deram entrada no SINAN (número de casos de recidiva divido pelo número de casos notificados como hanseníase e multiplicando o quociente por 100).

Foi construído um banco de dados, com as variáveis trabalhadas, no programa de estatística SPSS 13.0.

\section{RESULTADOS}

No período de janeiro de 2000 a dezembro de 2005, foram analisados 142 casos suspeitos de recidiva, que entraram no SINAN, advindos de diversos municípios do Estado do Espírito Santo. Do total de notificações, foram excluídos 38 casos, por não se enquadrarem no diagnóstico de recidiva.

Durante o período do estudo, 104 casos foram enquadrados no diagnóstico de recidiva de hanseníase, determinando uma magnitude de 1,12\% de casos de recidiva. Esta foi calculada através do número de recidivas durante o período do estudo em relação ao número de casos novos de hanseníase.

Dentre os pacientes com recidiva, a idade mínima foi de 14 anos e a máxima de 91 anos, com mediana de 46 anos e seis meses; 59,6\% eram do sexo masculino e 40,4\% do sexo feminino; 42,3\% eram brancos, 43,3\% mulatos e 14,4\% negros.

$\mathrm{Na}$ avaliação do período de incubação, em 98 pacientes com recidiva, observou-se que um (1\%) paciente (IC95\% 0,9-2,96) multibacilar recidivou no primeiro ano após o tratamento inicial, 51 (52\%) pacientes (IC95\% 43-61) entre um e cinco anos (17 PB e $34 \mathrm{MB})$ e 46 (46,9\%) pacientes (IC95\% 37-56) após cinco anos (15 PB e $31 \mathrm{MB})$.

A distribuição dos pacientes quanto à forma clínica da doença no primeiro diagnóstico e na recidiva pode ser observada na Tabela 1. No primeiro diagnóstico de hanseníase, 37 pacientes apresentaram a forma indeterminada, 27 pacientes a tuberculóide, 14 pacientes a dimorfa e 26 pacientes a virchowiana. Na recidiva

\section{TABELA 1}

Distribuição dos 104 pacientes em relação às formas clínicas de hanseníase no primeiro diagnóstico e na rediciva.

\begin{tabular}{lrrrrrrr}
\hline & \multicolumn{3}{c}{$1^{\circ}$ diagnóstico } & & \multicolumn{3}{c}{ Recidiva } \\
\cline { 2 - 4 } \cline { 6 - 8 } Forma clínica & $\mathrm{n}^{0}$ & $\%$ & IC95\% & & $\mathrm{n}^{0}$ & $\%$ & IC95\% \\
\hline Indeterminada & 37 & 35,5 & $26,5-44,5$ & & 07 & 6,7 & $1,8-11,6$ \\
Tuberculóide & 27 & 26,0 & $17,4-34,5$ & & 28 & 26,9 & $18,2-35,5$ \\
Dimorfa & 14 & 13,5 & $6,8-20,1$ & & 35 & 33,7 & $24,5-42,8$ \\
Virchowiana & 26 & 25,0 & $16,5-33,4$ & & 34 & 32,7 & $23,6-41,8$ \\
\hline Total & 104 & 100,0 & & 104 & 100,0 \\
\hline
\end{tabular}

IC95\%: intervalo de confiança 95\%. 
da hanseníase, sete pacientes tiveram a forma indeterminada, 28 pacientes a tuberculóide, 35 pacientes a dimorfa e 34 pacientes a virchowiana.

As frequências dos pacientes em relação ao número de lesões de pele e de troncos nervosos afetados em relação às formas clínicas na recidiva da doença se encontram na Tabela 2. Dois pacientes com a forma tuberculóide, dois com a dimorfa e três com a virchowiana não tinham lesões cutâneas. Nestes casos, o diagnóstico de recidiva se deveu a presença de neurite e/ou alteração no trajeto de nervos periféricos, que não existiam no primeiro diagnóstico, associado ao período de incubação acima de três anos da alta por cura do aparecimento destas alterações neurais, ou falta de informação do número de lesões nos prontuários pesquisados. Nos sete pacientes com a forma indeterminada na recidiva, o número de lesões cutâneas variou de uma a seis lesões e nenhum tronco nervoso afetado. 0 s 26 pacientes com a forma tuberculóide apresentaram de uma a cinco lesões cutâneas e a maioria (17/26) não tinha alteração nos nervos periféricos. Dos 35 pacientes com a hanseníase dimorfa, 24 deles apresentaram de uma a cinco lesões cutâneas e 16 pacientes tinham mais de um tronco nervoso acometido. Dos 34 pacientes com a forma virchowiana 14 tinha até cinco lesões na pele, 11 mais de cinco lesões, 16 pacientes não tinham os nervos periféricos afetados e 12 pacientes apresentavam mais de um nervo acometido.

\section{TABELA 2}

Distribuição do número de lesões cutâneas e de nervos afetados em 104 pacientes diagnosticados como recidiva de hanseníase, de acordo com a forma clínica da doença na recidiva.

\begin{tabular}{|c|c|c|c|c|c|c|c|c|}
\hline \multirow[b]{2}{*}{ Forma Clínica } & \multicolumn{4}{|c|}{ Lesões cutâneas } & \multicolumn{4}{|c|}{ Nervos afetados } \\
\hline & 0 & $0-5$ & $>5$ & $\begin{array}{c}\text { sem } \\
\text { registro }\end{array}$ & nenhum & 1 & $>1$ & $\begin{array}{c}\text { não } \\
\text { avaliado }\end{array}$ \\
\hline Indeterminada & - & 6 & 1 & - & 7 & - & - & - \\
\hline Tuberculóide & 2 & 26 & - & - & 17 & 5 & 5 & 1 \\
\hline Dimorfa & 2 & 24 & 8 & 1 & 14 & 3 & 16 & 2 \\
\hline Virchowiana & 3 & 14 & 11 & 6 & 16 & 2 & 12 & 4 \\
\hline Total & 7 & 70 & 20 & 7 & 54 & 10 & 33 & 7 \\
\hline
\end{tabular}

Dos 95 pacientes com recidiva que realizaram baciloscopias, 55 (57,9\%) pacientes (IC95\% 47,8-67,8) apresentaram baciloscopias negativas e $40(42,1 \%)$ pacientes (IC95\% 32,2$51,9)$ baciloscopias positivas, com bacilos íntegros. Vinte e quatro pacientes embora apresentassem baciloscopias negativas, foram considerados clinicamente como hanseníase dimorfa-dimorfa (18 casos) e hanseníase virchowiana (seis casos), e, para fins de tratamento como multibacilares, seguindo-se a recomendação do Ministério da Saúde, orientando que os pacientes com cinco ou mais lesões cutâneas devem receber a PQT/MB.

A avaliação do grau de incapacidade na recidiva revelou que: $66(68 \%)$ pacientes (IC95\% 58,6-77,4) tinham grau zero, 25 (25,8\%) pacientes (IC95\% 17,6-34,3) grau I e seis (6,2\%) pacientes (IC95\% 1,4-10,8) grau II.

0 desenvolvimento de estados reacionais durante e após o primeiro tratamento estava notificado em 39 prontuários. Verificou-se que $30(76,9 \%)$ pacientes (IC95\% 62,5-91,3) não desenvolveram nenhuma reação e nove $(23,1 \%)$ pacientes (IC95\% 14,7-32,6) apresentaram reações tipo I e/ou II.

A informação sobre o convívio com algum contato que tivesse adoecido de hanseníase após o término do tratamento do paciente constava em 25 prontuários, sendo que apenas um (4\%) paciente (IC95\% 0,1-7,9) teve convívio intradomiciliar com um parente que desenvolveu a hanseníase.

Os tratamentos recomendados aos pacientes no primeiro diagnóstico e na recidiva podem ser observados na Tabela 3 . Na primeira terapêutica, 52 pacientes foram tratados com o esquema $\mathrm{PB}$ adotado pelo Ministério da Saúde, que consiste na dose mensal de rifampicina $600 \mathrm{mg}$ associada a dapsona $100 \mathrm{mg}$ por dia, num total de seis doses, administradas em até nove meses. Este esquema foi administrado em 25 pacientes na recidiva da doença. Trinta e um pacientes receberam a PQT/MB no primeiro diagnóstico, ou seja, rifampicina $600 \mathrm{mg}$ e clofazimina $300 \mathrm{mg}$ como dose mensal e dapsona $100 \mathrm{mg}$ e clofazimina $50 \mathrm{mg}$ por dia, num total de 24 doses, administradas em até 36 meses. A maioria (78/104) dos

TABELA 3

Distribuição dos pacientes hansenianos em relação à terapêutica adotada no primeiro diagnóstico e na recidiva.

\begin{tabular}{lrrrrrrrr}
\hline & \multicolumn{3}{c}{$1^{\circ}$ diagnóstico } & & \multicolumn{3}{c}{ Recidiva } \\
\cline { 2 - 4 } \cline { 6 - 8 } Terapêutica & $n^{0}$ & $\%$ & IC95\% & & $n^{0}$ & $\%$ & IC95\% \\
\hline PB/MS & 52 & 52,5 & $42,6-62,3$ & & 25 & 24,0 & $15,6-32,3$ \\
MB/MS & 31 & 31,3 & $22,1-40,4$ & & 78 & 75,0 & $67,0-82,9$ \\
Alternativo & 7 & 7,0 & $2,0-12,0$ & & 1 & 1,0 & $0,9-2,91$ \\
ROM (dose única) & 5 & 5,1 & $0,7-9,34$ & & - & & - \\
Monoterapia(dapsona) & 4 & 4,1 & $0,2-7,9$ & & - & & - \\
\hline Total & 94 & 100,0 & & & 104 & 100,0 &
\end{tabular}

PB: paucibacilares, MB: multibacilares, ROM: rifampicina, ofloxacina e minociclina.

pacientes que recidivou foi retratada com o esquema PQT/MB. 0 tratamento alternativo utilizado em sete pacientes no primeiro diagnóstico e em um paciente com recidiva foi devido a efeitos colaterais a dapsona, que foi substituída pela clofazimina no esquema PB e ofloxacina 400mg por dia no MB. Cinco pacientes que apresentaram lesão única, nenhum tronco nervoso afetado e baciloscopia negativa no primeiro diagnóstico foram tratados com o esquema de dose única ROM (rifampicina $600 \mathrm{mg}$, ofloxacina 400mg e minociclina 100mg). Quatro pacientes diagnosticados e tratados antes da introdução da PQT em 1986 no Brasil haviam recebido rifampicina $600 \mathrm{mg}$ por dia por três meses associada à dapsona 100mg por dia, que era utilizada durante anos, até regressão clínica e baciloscópica. Ao final do tratamento da recidiva todos os casos receberam alta por cura.

\section{DISCUSSÃo}

A magnitude da recidiva de hanseníase, durante o período do estudo, demonstrou ser baixa $(1,12 \%)$, corroborando os estudos de alguns autores brasileiros que obtiveram as taxas de recidiva de hanseníase, tais como: Marója e cols ${ }^{13}$ que encontraram taxa de $0,95 \%$ em análise retrospectiva de 1982 a 2001 no Amazonas, Nery e cols ${ }^{15}$ que observaram taxa de $0,18 \%$ num estudo com 1.584 
pacientes tratados pela PQT entre 1986 a 2000 e Hércules ${ }^{10}$ que encontrou taxa bruta de recidiva de $0,64 \%$, no período de 1990 a 2003, em Duque de Caxias no Rio de Janeiro.

0 período de incubação da recidiva mostrou que em torno da metade dos pacientes recidivou entre um e cinco anos e os demais acima de cinco anos da alta do primeiro diagnóstico. A recidiva precoce, na qual o período de incubação é pequeno, geralmente, deve-se à terapia inadequada, seja por irregularidade terapêutica, abandono do tratamento ou erro de classificação. Nesses casos, não deveria ser aplicado o diagnóstico de recidiva. A recidiva tardia pode ocorrer devido à reativação de bacilos persistentes ou à reinfecção, que não podem ser confundidas com a reação da hanseníase ${ }^{7}$.

As formas clínicas mais freqüentes foram a dimorfa e a virchowiana, nem sempre acompanhadas de positividade nas baciloscopias, cujas explicações podem estar ligadas a fatores operacionais em uma das etapas do exame (coleta, coloração e leitura) ou mesmo ser devido ao diagnóstico precoce da recidiva, especialmente na forma dimorfa. A decisão de tratá-los como MB deveu-se ao número de lesões (acima de cinco) e/ou à quantidade de troncos nervosos afetados (acima de um), além de serem consideradas as possibilidades de falhas técnicas na realização das baciloscopias. Pandian e cols ${ }^{18}$ estudaram 263 pacientes com recidiva, sendo 130 casos da forma dimorfa. Gelber e cols ${ }^{6}$ descreveram 22 casos de recidiva, sendo dez da forma dimorfatuberculóide e 12 da virchowiana.

Nas fichas de sete pacientes com as formas clínicas tuberculóide, dimorfa e virchowiana constavam que não apresentavam lesões cutâneas. Estes dados podem ser devido a formas neurais puras, erro de digitação das fichas do SINAN ou falta de informação nos prontuários.

No estudo, observou-se maior frequiência de pacientes com até cinco lesões e nenhum tronco nervoso afetado, refletindo ou a ausência de incapacidade física instalada, demonstrando que a procura pela assistência médica foi mais precoce ou falhas na avaliação neurológica do paciente.

Quanto à baciloscopia na recidiva, alguns pacientes foram negativos no primeiro diagnóstico e recidivaram com positividade neste exame, denotando evolução multibacilar de algumas formas clínicas e/ou terapia insuficiente na eliminação do bacilo. Li e cols $^{12}$ estudaram 8.307 pacientes tratados com a PQT de 1986 a 1995 e detectaram 16 recidivas, sendo que dos onze casos inicialmente $\mathrm{PB}$, três recidivaram com a hanseníase dimorfatuberculóide e os demais como MB. Haldar e cols ${ }^{9}$ diagnosticaram 128 casos de recidiva, sendo que dos 102 casos PB, 94 casos permaneceram neste espectro.

Brito e cols ${ }^{2}$ observaram que $33,9 \%$ dos casos, denominados pela autora de retratamentos, apresentavam comunicantes portadores de hanseníase e sugeriram a possibilidade de reinfecção, levando-se em consideração a irreversibilidade da deficiência imunológica específica ao Mycobacterium leprae. № estudo, não foi possível verificar se os pacientes tiveram contato com comunicantes que adoeceram após sua alta terapêutica, por falta desta informação na maioria dos prontuários enviados ao Centro de Referência. Como também, se o paciente apresentou reação após o primeiro tratamento, portanto, se utilizou drogas imunossupressoras, como os corticosteróides, a dose e o tempo, o que poderia contribuir para o reaparecimento da doença, em decorrência da alteração imunológica poder facilitar a reativação de bacilos persistentes. Gebre e cols 5 não observaram associação do uso de corticosteróide sistêmico e a recidiva em 502 pacientes acompanhados por oito anos. Brito em 2004 estudou 310 pacientes com hanseníase, e os que apresentaram episódios reacionais tinham aproximadamente três vezes mais possibilidade de recidiva ${ }^{1}$.

0 tratamento mais utilizado na recidiva foi a $\mathrm{PQT} / \mathrm{MB}$, apesar de $57,8 \%$ dos pacientes apresentarem baciloscopias negativas, sendo, portanto, a forma terapêutica escolhida baseada no número de lesões e troncos nervosos afetados, como também, na segurança da terapêutica utilizando-se três drogas.

0 estudo foi realizado com dados obtidos de prontuários de pacientes atendidos nas Unidades Básicas de Saúde. Estes prontuários são preenchidos por médicos clínicos gerais ou do Programa de Saúde da Família. Esta é uma das limitações do estudo, pois, o preenchimento das notificações é passível de falha.

Para que seja conhecida a real situação de recidiva da hanseníase nos Estados brasileiros, se faz necessário maior número de estudos sobre o tema. Para tanto, diante de um caso suspeito de recidiva, este deveria ser enviado a um Centro de Referência para a verificação detalhada de todos os aspectos clínicos, epidemiológicos e laboratoriais, que pudessem confirmar o diagnóstico de recidiva. Não menos importante é a avaliação da resistência medicamentosa às drogas dapsona e rifampcina, cuja vigilância já é recomendada pela $\mathrm{OMS}^{8}$.

\section{REFERÊNCIAS}

1. Brito MFM. O retratamento em hanseníase: Identificação dos fatores de risco - Um estudo caso-controle. Tese de Mestrado, Universidade Federal de Pernambuco, Recife, PE, 2004.

2. Brito MFM, Gallo MEN, Ximenes RAA. 0 retratamento por recidiva em hanseníase. Anais Brasileiros de Dermatologia 80: 255-260, 2005.

3. Daumerie D, Pannikar V. Issues in evaluating information on relapse in leprosy. Indian Journal of Leprosy 67: 27-33, 1995

4. Ganapati R, Bulchand H, Pai VV, Kingsley S, Revankar CR. Relapsing multibacillary leprosy - a new dimension to transmission in urban areas. International Journal of Leprosy 69: 114-115, 2001.

5. Gebre S, Paul S, Peter B. Relapses after fixed duration multiple drug therapy: the AMFES cohort. Leprosy Review 71: 325-331, 2000.

6. Gelber RH, Balagon MV, Cellona RV. The relapse rate in MB leprosy patients treated with 2-years of WHO-MDT is not low. International Journal of Leprosy 72: 493-500, 2004.

7. Girdhar Bk, Girdhar A, Kumar A. Relapses in multibacillary leprosy patients: effect of length of therapy. Leprosy Review 71: 144-153, 2000.

8. Guidelines for global surveillance of drug resistance in leprosy. New Delhi, World Health Organization, Regional Offi ce for South-East Asia, 2009 (WHOSEAGLP-2009.

9. Haldar A, Mahapatra BS, Mundle M, Haldar S, Saha AK. A study of relapse after MDT in a district in West Bengal, India. Indian Journal of Leprosy 75: 1-8, 2003.

10. Hércules FM. Recidiva de hanseníase no município de Duque de Caxias. Dissertação de mestrado - Dermatologia. Universidade Federal do Rio de Janeiro, Rio de Janeiro, RJ, 2004. 
11. Klioze AM, Ramos-Caro FA. Visceral leprosy. International Journal of Dermatology 39: 641-658, 2000

12. Li HY, Hu LF, Huang WB, Liu GC, Yuan LC, Jin Z, Li X, Li JL, Yang ZM. Risk of relapses in leprosy after fixed-duration multidrug therapy. International Journal of Leprosy 65: 238-245, 1997.

13. Marója MF, Pedrosa V, Pereira ES, Berbegal MDP, Cunha MGS, Schettini AP. Hansen's disease relapse in the control programme of Amazonas state. In: Abstract $16^{\text {th }}$ International Leprosy Congress, Salvador, Bahia, p.154, 2002

14. Ministério da Saúde. Legislação sobre o controle da hanseníase no Brasil: Área Técnica de Dermatologia Sanitária, Brasília, p.48, 2000.

15. Nery JAC, Lopes ACS, Albuquerque ECA, Machado AM, Gallo MEN. Hanseníase: recidivas pós poliquimioterapia com duração fixa. $I n$ : $16^{\text {th }}$ International Leprosy Congress, Salvador, Bahia, p.159, 2002
16. Norman G, Joseph G, Richard J. Relapses in multibacillary patients treated with multidrug therapy until smear negativity: findings after twenty years. International Journal of Leprosy 72: 1-7, 2004

17. Oliveira MLW. A cura da hanseníase X magnitude das recidivas. Anais Brasileiros de Dermatologia 72: 63-69, 1997.

18. Pandian TD, Sitambaram M, Bharati R, Ramu G. Relapses in non-lepromatous and intermediate forms of leprosy. Indian Journal of Leprosy 57: 149-158, 1983.

19. Secretaria de Estado da Saúde. Programa Estadual de Controle da Hanseníase. Hanseníase - dados epidemiológicos 2005. Espírito Santo: Superintendência de Planejamento Epidemiologia e Informação, p. 22-37, 2005.

20. You EY, Kang TJ, Kim SK, Lu SB, Chae GT. Mutations in genes related to drug resistance in Mycobacterium leprae isolates from leprosy patients in Korea. Journal of Infection 50: 6-11, 2005. 